\title{
A new species of Daphne (Thymelaeaceae): D. jejudoensis M. Kim
}

\author{
Jungsim Lee, Kang-Hyup Lee', Soonku So, Changhak Choi and Muyeol Kim* \\ Department of Biological Sciences, Chonbuk National University, Jeonju 561-756, Korea \\ ${ }^{1}$ Pyunggang Botanical Garden, Pochen 487-892, Korea \\ (Received 1 May 2013; Revised 31 May 2013; Accepted 11 June 2013)

\section{팥꽃나무속(팥꽃나무과)의 신종: 제주백서향 (Daphne jejudoensis M. Kim)} \\ 이정심 · 이강협 ${ }^{1} \cdot$ 소순구 · 최창학 · 김무열* \\ 전북대학교 자연과학대학 생명과학과, ${ }^{1 ㅍ ㅕ ㅇ ㄱ ㅏ ㅇ ㅅ ㅣ ㄱ ㅁ ㅜ ㄹ ㅇ ㅝ ㄴ ~}$
}

\begin{abstract}
A new species, Daphne jejudoensis M. Kim, has been named and described from Seonheul, Jejudo Province, Korea. Daphne jejudoensis shares several characteristics (white calyx colors, long calyx tube, evergreen leaves, leathery leaf textures, etc.) with its related species D. kiusiana, but it is distinct from D. kiusiana which has a hairy calyx tube and lobes, short calyx lobes, oblanceolate leaves (with acute apex), and a different distribution (coastal region in Geojedo Is.) by having a glabrous calyx tube and lobes, long calyx lobes, elliptic leaves (with acuminate apex), and by its distribution (inland region in Jejudo Is.).
\end{abstract}

Keywords: Daphne jejudoensis, Daphne kiusiana, Thymelaeaceae

적 요: 제주도 선흘에서 팥꽃나무속의 신종인 제주백서향(Daphne jejudoensis M. Kim)을 발견하여 기재하 였다. 제주백서향은 꽃이 백색이고 잎은 상록성이며 긴 꽃받침 통을 가져 백서향(D. kiusiana)과 유사하다. 그 러나 제주백서향은 꽃받침 통과 열편에 털이 없고 장타원형(점첨두) 잎을 가지며 제주도의 중산간지역에서 자라는 반면에, 백서향은 꽃받침 통과 열편에 털이 있고 도피침형(급첨두) 잎을 가지며 거제도의 해안가에서 자라는 점에서 두 종이 뚜렷하게 차이가 난다.

주요어: 제주백서향, 백서향, 팥꽃나무과

The genus Daphne L. (Thymelaeaceae) consists of approximately 95 species and is distributed primarily in regions of the SE Asia including China, Korea, and Japan, and also Europe (Hong, 2007; Wang et al., 2007). The genus Daphne are characterized by shrubs which are $0.5-2 \mathrm{~m}$ tall, 8 stamens, headlike short raceme inflorescence, and drupe fruits (Hong, 2007). Four taxa of Daphne are distributed in Korea (Lee, 1980; Lee, 1996; Lee, 2006; Hong, 2007). Of the four taxa, Daphne genkwa Siebold \& Zucc. occurs near the seashore of southern

*Author for correspondence: mykim@jbnu.ac.kr

http://www.pltaxa.or.kr

Copyright (C) 2013 the Korean Society of Plant Taxonomists areas in Korea, while D. kiusiana Miq. is rather rare and sporadically distributed in Korea (Geojedo Is.), Japan, and China. A new species from Seonheul, Andeog, and Mureung in Jejudo Province, Korea was discovered. Until now, this new species was misidentified as Daphne kiusiana Miq. (Chung, 1957; Lee, 1980; Lee, 1996; Lee, 2006). In this paper, we named this new taxon Daphne jejudoensis M. Kim and described several morphological features relative to its related species D. kiusiana and D. odora Thunb. The new species shares several characteristics with its related species, but it can be distinguished based on calyx surface (i.e. glabrous), calyx tube (i.e., long size), calyx lobes (i.e., long size), leaf shape (i.e., elliptic), distribution (i.e., Jejudo Is.), etc. 
Daphne jejudoensis M. Kim, sp. nov. (Figs. 1-3) Korean name: Jeju-Baek-Seohyang 제주백서향

Shrubs evergreen, $60-90 \mathrm{~cm}$ tall. Branches purplish brown, slender, glabrous. Leaves alternate; petiole 2-3 mm, glabrous; leaf blade elliptic, 7.0-8.5 × 1.7-2.5 cm, leathery, both surface glabrous, base cuneate, apex acuminate; veins 6-8 pairs. Inflorescence terminal, headlike short raceme, 12-16 flowered;

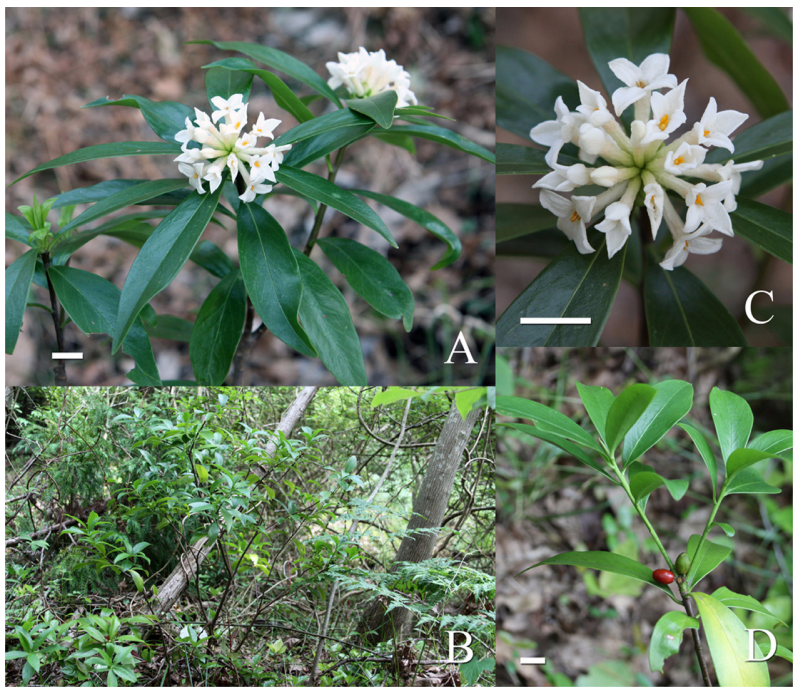

Fig. 1. Photographs of Daphne jejudoensis M. Kim at the type locality. A. Branches with flowers; B. Habitat; C. Flower; D. Fruit. Scale bars $1 \mathrm{~cm}$ in $\mathrm{A}, \mathrm{C}$ and $\mathrm{D}$.
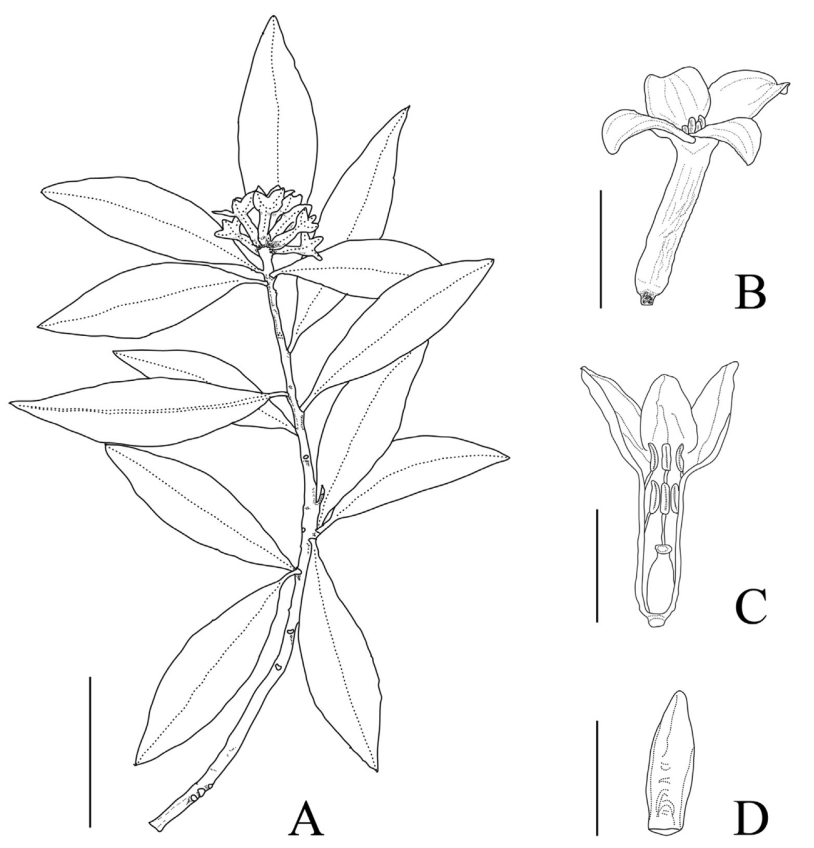

Fig. 2. Daphne jejudoensis M. Kim A. A branch with flowers; B. Flower; C. Inner view of flower; D. Bract. Scale bars $5 \mathrm{~cm}$ in A; 1 $\mathrm{cm}$ in $\mathrm{B}$ and $\mathrm{C} ; 0.5 \mathrm{~cm}$ in $\mathrm{D}$. bracts lanceolate 5-6 $\times 1.5-3.0 \mathrm{~mm}$, glabrous. Flowers bisexual, fragrant. Calyx white; tube $12-14 \mathrm{~mm}$, exterior glabrous; lobes 4, ovate, 6-7 mm, base auriculate, apex obtuse.s Petals absent. Stamens 8 , lower whorl inserted just above middle of calyx tube, upper whorl in throat; filaments 0.4-0.6 mm; anthers oblong, 2-3 $\mathrm{mm}$. Disk annular. Ovary oblong, glabrous; style short; stigma capitate. Drupe red, globose, 8-10 mm. Flowering March, fruit June.

Holotype: Seonheul, Jejudo Province, Korea. March 15, 2013. M. Kim 130201. Herbarium of Jeonbuk National University (JNU).

Isotype: Seonheul, Jejudo Province, Korea. March 15, 2013. M. Kim 130201 (5 sheets, JNU).

Paratype: Jejudo (Jocheon). Jan. 16, 1991. H. Kim (JNUB); Jejudo (Gujoa). Jan. 16, 1998. M. Moon (JNUB); Jejudo (Andeog). Mar. 7, 2002. Y. Kang (KB); Jejudo (Andeog). March 16, 2013. M. Kim 130207 (JNU); Jejudo (Mureung). March 16, 2013. M. Kim 130208 (JNU); Jejudo (Seonheul). June 3, 2013. M. Kim 130213 (JNU).

Distribution: Seonheul, Andeog, and Mureung in Jejudo Province, Korea.

Etymology: The specific epithet is derived from Jejudo Is. where this new species is located.

Habitats: Daphne jejudoensis M. Kim was discovered in

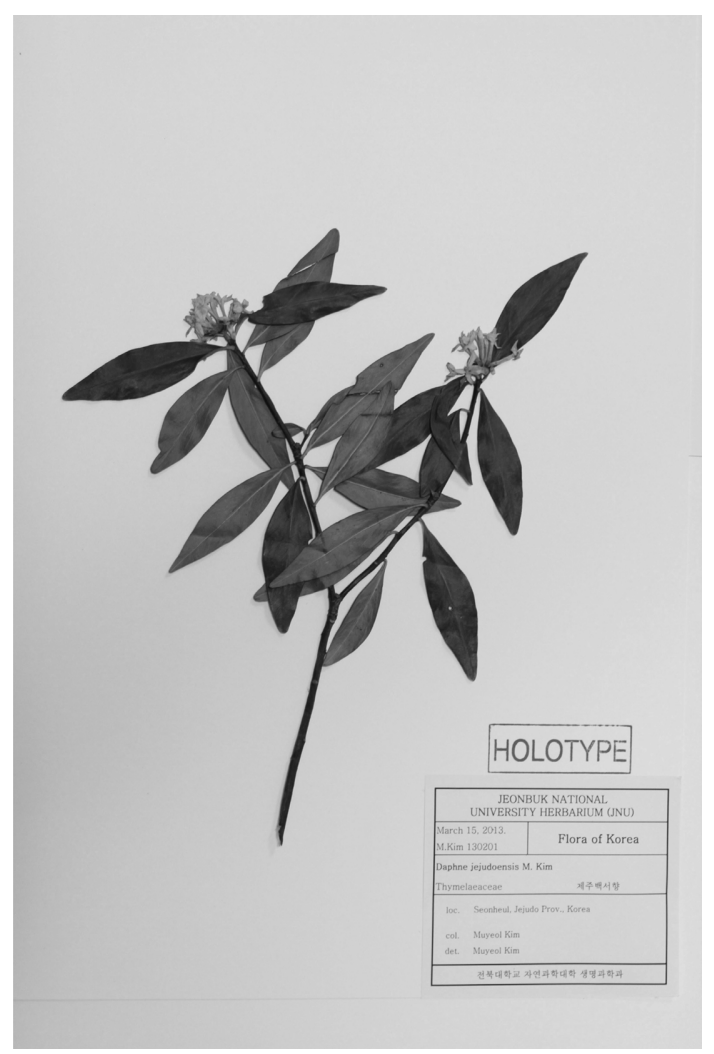

Fig. 3. Holotype of Daphne jejudoensis M. Kim 
Table 1. Comparison of morphological characters among Daphne jejudoensis M. Kim, D. kiusiana and D. odora.

\begin{tabular}{|c|c|c|c|}
\hline Characters & D. jejudoensis & D. kiusiana & D. odora \\
\hline Plant height & $60-90 \mathrm{~cm}$ & $50-120 \mathrm{~cm}$ & $70-90 \mathrm{~cm}$ \\
\hline Habitat & inland region & coastal region & . \\
\hline Twig & slender & slender & stout \\
\hline Twig internode & $5-10 \mathrm{~mm}$ & $1-5 \mathrm{~mm}$ & $5-20 \mathrm{~mm}$ \\
\hline Leaves on old branches & present & absent & absent \\
\hline Leaf shape & elliptic & oblanceolate & oblanceolate \\
\hline Leaf blade length & $7.0-8.5 \mathrm{~cm}$ & $7.5-9.0 \mathrm{~cm}$ & $6.5-8.0 \mathrm{~cm}$ \\
\hline Leaf blade width & $1.7-2.5 \mathrm{~cm}$ & $2.5-3.5 \mathrm{~cm}$ & $2.0-2.8 \mathrm{~cm}$ \\
\hline Leaf apex & acuminate & acute & acute \\
\hline Pedicel & glabrous & glabrous & pubescent \\
\hline Bract margin & glabrous & glabrous & pubescent \\
\hline Calyx color & white & white & pale red-purple \\
\hline Calyx exterior & glabrous & hairy & glabrous \\
\hline Calyx tube length & $12-14 \mathrm{~mm}$ & $8-10 \mathrm{~mm}$ & $8-9 \mathrm{~mm}$ \\
\hline Calyx lobe length & $6-7 \mathrm{~mm}$ & $3-4 \mathrm{~mm}$ & $6-8 \mathrm{~mm}$ \\
\hline Fruit length & $8-10 \mathrm{~mm}$ & $9-11 \mathrm{~mm}$ & . \\
\hline Distribution & Jejudo Is. & Geojedo Is. & cultivated \\
\hline
\end{tabular}

broad-leaved forest regions in Seonheul, Jejudo Province, Korea. The new species occurs rarely in evergreen woodlands dominated by Castanopsis cuspidata (Thunb.) Schottky, Machilus thunbergii Siebold \& Zucc., and Cudrania tricuspidata (Carriere) Bureau ex Lavallee. This species also lives with other herbaceous species dominated by Semiaquilegia adoxoides (DC.) Makino, Viola grypoceras A. Gray, and Dryopteris lacera (Thunb.) Kuntze.

\section{Key to Daphne taxa in Korea.}

1. Calyx exterior glabrous

2. Leaves deciduous; calyx tube 4-5 $\mathrm{mm}$; inflorescence ebracteate

.......... D. pseudomezereum var. koreana 두메닥나무

2. Leaves evergreen; calyx tube 8-14 mm; inflorescence bracteate

3. Calyx pale red-purple; calyx tube $8-9 \mathrm{~mm}$; leaves oblanceolate D. odora 서향

3. Calyx white; calyx tube $12-14 \mathrm{~mm}$; leaves elliptic

D. jejudoensis 제주백서향

1. Calyx exterior hairy

4. Calyx purple; leaves deciduous

4. Calyx white; leaves evergreen

D. genkwa 팥꽃나무

D. kiusiana 백서향
Populations of the new species, Daphne jejudoensis M. Kim were discovered at Seonheul, Andeog, and Mureung in Jejudo Province, Korea. Daphne jejudoensis shares several characteristics (white calyx colors, long calyx tube, evergreen leaves, leathery leaf textures, etc.) with its related species D. kiusiana Miq., but it is distinct from D. kiusiana which has a hairy calyx tube, short calyx lobes, oblanceolate leaves (with acute apex), and a different distribution (coastal region in Geojedo Is.) by having a glabrous calyx tube, long calyx lobes, elliptic leaves (with acuminate apex), and by its distribution (inland region in Jejudo Is.)(Table 1, Appendix). Thus, the authors described this taxon as a new species of the genus Daphne.

\section{Acknowledgement}

We are deeply indebted to an anonymous reviewer for improving our manuscript, and also to Won-Mun Jeong and Jeong-Hui Lee for their kindly help.

\section{Literature Cited}

Chung, T. H. 1957. Koran Flora, Part 1. Woody Plants. Shinjisa. Seoul. (in Korean)

Hong, S. P. 2007. Daphne L. In The Genera of Vascular Plants of Korea. Park, C.-W. (ed.), Academy Publishing Co., Seoul. Pp. 
633-634.

Lee, T. B. 1980. Illustrated Flora of Korea. Hyangmunsa, Seoul. (in Korean)

Lee, W. T. 1996. Lineamenta Florae Koreae. Academy Press. Seoul. (in Korean)
Lee, Y. N. 2006. New Flora of Korea. Kyohaksa, Seoul. (in Korean) Wang, Y., M. G. Gilbert, B. Mathew and C. D. Brichell. 2007. Daphne L. In Flora of China. Vol. 13. Wu, Z.-Y. and P. H. Raven (eds.). Science Press, Beijing and Missouri Botanical Garden Press, St. Louis. Pp. 230-245. 


\section{Appendix. Examined specimens of Daphne taxa in} Korea.

\section{D. jejudoensis M. Kim 제주백서향}

Jeju: Seonheul. Mar. 15, 2013. M. Kim 130201 (JNU); Andeog. Mar. 16, 2013. M. Kim 130207 (JNU); Mureung. Mar. 16, 2013. M. Kim 130208 (JNU); Seonheul. Jun. 3, 2013. M. Kim 130213 (JNU).

\section{D. kiusiana Miq. 백서향}

Kyeongnam: Geojedo Is. Mar. 30, 2013. M. Kim 130209 (JNU); Geojedo Is. Jun. 8, 2013. M. Kim 130214 (JNU).
D. odora Thunb. 서향

Kyeongnam: Geojedo Is. Mar. 30, 2013. M. Kim 130210 (JNU).

Jeonbuk: Jeonju. Feb. 20, 2013. M. Kim 130190 (JNU).

D. genkwa Siebold \& Zucc. 팥꽃나무

Jeonbuk: Byeonsan. Mar. 28, 2013. M. Kim 130211 (JNU).

D. pseudomezereum var. koreana (Nakai) Hamaya 두메 닥나무

Kangwon: Mt. Deoghang. Apr. 1, 2013. M. Kim 130212 (JNU). 